International Journal on Design \& Manufacturing Technologies, Vol. 8 No. 2 July 2014

\title{
IMPROVEMENT IN MOTION CHARACTERISTICS OF CAM-FOLLOWER SYSTEMS USING NURBS
}

\author{
N. Sateesh \\ Vijay College of Engineering for women, Nizamabad-503 003, A.P India. \\ Email: nagari_sateesh@yahoo.com
}

Absrtact.

This paper proposes an integrated system for improving the cam-follower performance. The design method is to improve the motion characteristics of a cam follower-system. Improvement of the motion characteristics is to achieve low maximum velocity and acceleration for each follower motion curve so that jerk is minimum. Representing basic follower motion curves with nonuniform rational B-splines (NURBS) can do this. Conventional methods in designing and manufacturing of cam are tedious and time consuming. Even programming them on a computer numerical control (CNC) machine can be a difficult job because of the complexity of the cam profiles. . A CAD/CAM system is developed, which provides useful design information such as graphical and numerical representation of displacement, velocity, acceleration, jerk, pressure angle and cam profiles for both basic curves and NURBS. An analysis can be carried out based on maximum velocities and accelerations to select the best cam follower motion. It also provides cam profile coordinates for basic curves and NURBS to manufacture a cam on CNC machines.

Key words: plate-cams, disc-cams, cam profile, NURBS, B-Spline,, computer numerical control (CNC), Computer aided design and computer aided manufacturing (CAD/CAM), R-D-R-D (rise-dwell-return-dwell)

\section{INTRODUCTION}

A plate-cam is a disc-cam, which is cut out of a piece of flat metal or plate and used to transform a rotary motion into a translating or oscillating motion to its follower. Applications of these cams found in packaging machines, wire-forming machines, internal combustion engines, mechanical and electronic computers. Requirements for high performance of such machinery demand efficient methods for the design and manufacture of cams. The purpose of this paper is to develop a seamless integration of design and manufacturing of plate cams. NURBS have been used to design and optimization of cam profiles. The design parameters will be used in the manufacturing module that generates the NC program required for the machining of the designed cam.

\section{BASIC FOLLOWER MOTIONS}

To analyze the action of a cam, it is necessary to study its displacement-time diagram and its associated velocity and acceleration curves. Some of the most common types of follower motion curves selected by cam designers for a typical cam follower system shown in the Figure1 are:

1. Simple harmonic motion (S.H.M.).

2. 2 Cycloidal motion.

3. .Parabolic motion.

4. 3-4-5 polynomial.

5. 4-5-6-7 polynomial

6. Modified harmonic motion.

Simple harmonic motion has smoothness in velocity and acceleration during the stroke is advantage inherent in the curve. However, the instantaneous changes in the acceleration at the beginning and end of the stroke tend to cause vibration, noise, and wear. It is, therefore, suitable only for cams at medium or low speed. Cycloidal motion is obtained by rolling a circle on a straight line. It has the smoothest motion among all of the basic curves. The maximum value of the acceleration of the follower for a given rise is somewhat higher than that of the simple harmonic motion. Cycloidal curve is used often as a basis 
for designing cams for high-speed machinery because it results in low noise, vibration, and wear. Parabolic motion has constant acceleration and retardation following a parabolic equation. The 4-5-6-7 polynomial curve is worked out from a 7 th degree polynomial. It has good acceleration characteristics and is used for high-speed cams.

\section{A. Follower motion equations:}

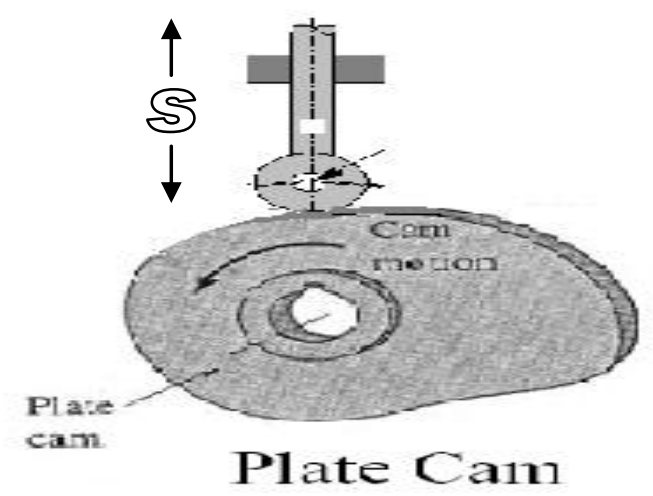

Displacement (S) equations for different follower motions against cam rotation angle $\theta$ have been presented as follows

Fig.1: Plate Cam

Simple harmonic: $S=h / 2[1-\cos (\Pi \theta / \beta)]$

Cycloidal: $\quad S=h / 2\left[\theta / \beta-\cos \left(2 \prod \theta / \beta\right)\right]$

Parabolic motion: $S=2 h[\theta / \beta]^{2}$

3-4-5 polynomial: $S=h\left[10\left((\theta / \beta)^{3}-15\left((\theta / \beta)^{4}+6(\theta / \beta)^{5}\right]\right.\right.$

4-5-6-7 polynomial $S=h \quad\left[35(\theta / \beta)^{4} \vdash-84\left((\theta / \beta)^{5}\right.\right.$ $\left.+70(\theta / \beta)^{6}-20(\theta / \beta)^{7}\right]$

Modified harmonic: $S=h / 2[(1-\cos (\theta / \beta)-0.25(1-\cos$ $(2 \theta / \beta))]$

Where $h$ is lift of the follower, $\beta$ is the angle of rise or return

\section{B. Cam profiles:}

The workout of a cam profile requires the drawing of many positions of the cam with the follower in each case in its related location. Depends up on the kind of follower, cams used with radial translating follower, an offset roller follower, flat face follower and oscillating followers. In radial translating follower centerline of the follower-stem passes through the center of the camshaft. In offset translating roller follower, the follower is offset from the camshaft center. Generally flat face follower perpendicular to the follower stem. Some times centerline of the flat face is offset from the centerline of the camshaft. In oscillating roller follower, follower oscillates about a pivot point depend upon the rotation of cam.

\section{Cam profile calculations:}

The following equations give points on the pitch curve of the cam and points on the cam profile itself in rectangular coordinates for roller follower which is in contact with the cam. The rectangular coordinates $(X, Y)$ about cam center for each angle of cam rotation $(\theta)$ as follows.

\section{Radial translating follower:}

$X=(R p+S) \sin \theta ; \quad Y=(R p+S) \cos \theta$

\section{NON-UNIFORM RATIONAL B-SPLINES (NURBS)}

A. Optimization of follower motion curves using NURBS:

The motion characteristics of the cam follower mechanisms can be improved by reducing the jerk. The jerk causes vibration, more contact stresses, wear and tear in the cam. This is predominant at the transition points i.e. points where follower motion changes from dwell to rise, rise to dwell, dwell to fall and fall to dwell etc. One method of improving the follower motion characteristics would be to represent the basic curves by Non Uniform Rational B-Splines (NURBS).

\section{B. NURBS:}

One of the most versatile tools for modeling curves is the Non-Uniform Rational B-splines (NURBS). It has been widely used in modeling of curves and surfaces in CAD/CAM as a standard. It is a smooth spline and it is a ratio of two non-rational B-spline basis functions, making it a vector- valued piecewise rational polynomial. NURBS 
offer a common mathematical form for representation and used for designing standard curves (conic and quadrics, etc), free form curves and surfaces. NURBS are invariant under translation, rotation, scaling, shear, and parallel and perspective projection. They have ability to interpolate or approximate a set of given data points. They provide local control of the curve shape as opposed to global control by using a special set of blending functions that provide local influence. Another advantage of NURBS is that they provide the ability to add control points without increasing degree of the curve. NURBS are free form curves having $\mathrm{C}^{0}$ (points continuity), $\mathrm{C}^{1}$ (slope continuity) and $\mathrm{C}^{2}$ (curvature continuity).

Mathematically NURBS can be defined by $n 1$ control points

$$
p(u)=\sum_{i=0}^{n} N_{i, k}(u) p_{i} h_{i} / \sum_{i=0}^{n} N_{i, k}(u) h_{i}
$$$$
0<=\mathrm{u}<\mathrm{u}_{\max }
$$

Where $p_{i}$ is a control point, $N_{i, k}(u)$ is a blending function, which is recursive in nature and polynomial of degree $k-1, h_{i}$ is weight at each control point and varies from 0 to 1 . If weights are equal at all control points than NURBS becomes non-rational B-splines. $p(u)$ is the position on the curve at parameter $u$. The range of parameter $u$ depends on the number of control points $n+1$ and the choice for $k$, so that $u$ varies from 0 to $n-k+2$.

The Blending function has the property of recursion, which is defined as

$N_{i, k}(u)=\left[\left(u-u_{i}\right) N_{i, k-1}(u)\right] /\left[u_{i+k-1}-u_{i}\right]+$
$\left[\left(u_{i+k}-u\right) N_{i+1, k-1}(u)\right] /\left[u_{i+k}-u_{i+1}\right]$

$N_{i, 1}(u)=1$ if $u_{i}<u<u_{i+1}, N_{i, 1}(u)=0$ otherwise -----(3)

Where $k$ controls the degree $(k-1)$ of the resulting polynomial in $u$ and also controls the continuity of the curve. The values $u_{i}$ are called knot values. They relate the parametric variable $u$ and control points $\left(p_{i}\right)$. The knot values $u_{i}$ are given by $u_{j}=0$ if $j<k, \quad u_{j}=\mathrm{j}-k+1 \quad$ if $k<=\mathrm{j}<=n, u_{j}=n-k+2 \quad$ if $\mathrm{j}>$ $n$ with $0<=j<=n+k$.

Number of knot values $(m)=n+k+1$,

i.e. $\quad u_{i}=\left[u_{0}, u_{1}, u_{2}, u_{3}, \ldots \ldots \ldots \ldots . ., u_{n+k}\right]-------(4)$

They are three ways to modify the shape of NURBS. Change the knot vector, move the control points or change the weights. It is relatively difficult to determine how a curve will respond to changes in the knot vector, this is not the best way to change curve shape. On the other hand, effect of changing a control point is predictable and intuitive. If a weight $h_{i}$ is increased or decreased in value, then the curve is pulled towards or pushed away from the respective control point $p_{i}$. This is the best way to modify the shape of the NURBS.

\section{Applying a NURBS approximation to a basic curve:}

In cam design, once the basic follower motion curve has been specified, it can then be approximated by NURBS. In the design of NURBS, the curve with degree $(k-1)$ three and the six control points $(n+1)$ of the basic curve are considered. The following steps illustrate the procedure by a simple example.

1.Create the basic curve of the selected follower motion (simple harmonic, cycloidal, etc).

2. Divide the angle of interval (rise or return) of the curve into 5 parts. Each part has the same angle of interval. This will create 6 control points, $p_{0}, p_{1}, p_{2}, p_{3}, p_{4}$, and $p_{5}$ lying on the curve.

3. These 6 control points are interpolated by considering the constraints of $n=5$ and $k=4$ in to the equations $1,2,3$ and 4 . The parametric equations obtained are:

$p_{x, y}=\left\{(1-u)^{3} p_{0} h_{0}+\left[u(1-u)^{2}+1 / 2(2-u)\left(-3 / 2 u^{2}+2 u\right)\right]\right.$ $\left.p_{1} h_{1}+\left[u / 2\left(-3 / 2 u^{2}+2 u\right)+u^{2} / 6(3-u)\right] p_{2} h_{2}+u^{3} / 6 p_{3} h_{3}\right\} /$ $\left\{(1-u)^{3} h_{0}+\left[u(1-u)^{2}+1 / 2(2-u)\left(-3 / 2 u^{2}+2 u\right)\right] h_{1}+[u / 2(-\right.$ $\left.\left.\left.3 / 2 u^{2}+2 u\right)+u^{2} / 6(3-u)\right] h_{2}+u^{3} / 6 h_{3}\right\}$

if $0<=u<1$

$p_{x, y}=\left\{1 / 4(2-u)^{3} p_{1} h_{1}+\left[u / 4(2-u)^{2}+(3-u) / 3\left(-u^{2}+3 u-3 / 2\right)\right] p_{2} h_{2}\right.$ $\left.+\left[u / 3\left(-u^{2}+3 u-3 / 2\right)+1 / 4(3-u)(u-1)^{2}\right] p_{3} h_{3}+1 / 4(u-1)^{3} p_{4} h_{4}\right\}$ $/\left\{1 / 4(2-u)^{3} h_{1}+\left[u / 4(2-u)^{2}+(3-u) / 3\left(-u^{2}+3 u-3 / 2\right)\right] h_{2}+[u / 3(-\right.$ $\left.\left.\left.u^{2}+3 u-3 / 2\right)+1 / 4(3-u)(u-1)^{2}\right] h_{3}+1 / 4(u-1)^{3} h_{4}\right\}$ 


$$
\text { if } 1<=u<2
$$

$p_{x, y}=\left\{1 / 6(3-u)^{3} p_{2} h_{2}+\left[u / 6(3-u)^{2}+(3-u) / 2\left(-3 / 2 u^{2}+7 u-\right.\right.\right.$

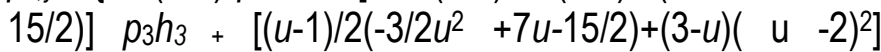
$\left.p_{4} h_{4}+(u-2)^{3} p_{5} h_{5}\right\} / 1 / 6(3-u)^{3} h_{2}+\left[u / 6(3-u)^{2}+(3-u) / 2\left(-3 / 2 u^{2}\right.\right.$ $+7 u-15 / 2)] h_{3}+\left[(u-1) / 2\left(-3 / 2 u^{2}+7 u-15 / 2\right)+(3-u)(u-2)^{2}\right]$ $\left.h_{4}+(u-2)^{3} h_{5}\right\}$

$$
\text { if } 2<=u<3
$$

Where $p_{x}$ is the $x$-coordinate point and $p_{y}$ is the $y$ coordinate point on the curve. Using the above procedure, each motion curve is converted into an equivalent NURBS. The new curve may look approximately the same as the former basic curve but it will have differences in the smoothness and maximum velocity and acceleration. These are the factors that affect the kinematics performance of the cam.

\section{INTEGRATED SYSTEM}

An integrated system for a disc-cam presented in this paper is an interactive computer program written in Visual Basic language. It is an user interactive system where user can design and manufacture the cam of his choice by providing necessary data such as type of basic follower motion, speed of cam (r.p.m.), lift of the follower (h) in $\mathrm{mm}$, and angle of rise, return, dwell (degrees).

Then the system computes the cam profile for the specified follower motion and generates the following information:

1.Graphical and numerical display of displacement, velocity, acceleration and jerk diagrams for each follower motion basic curves and approximated NURBS against cam rotation $\theta$.

2. Graphical and numerical display of cam profiles and cutter location data points of each follower motion basic curves and approximated NURBS against cam rotation $\theta$.

3. Numerical values of maximum acceleration and maximum velocity for each follower motion basic curves and approximated NURBS.
Numerical illustration: The integrated system for design and manufacturing of cams has been thoroughly tested for a typical input to the program, which is given in Figure 2.

Lift $=30 \mathrm{~mm}$, Rise angle $=60$ degrees, Dwell1 $=120$ Degrees, Return $=60$ Degrees, Dwell $2=120$ degrees, Cam speed $=20$ Rpm. Weights for NURBS h0 $=0.1$, $\mathrm{h} 1=0.2, \mathrm{~h} 2=0.3, \mathrm{~h} 3=0.4, \mathrm{~h} 4=0.5$ and $\mathrm{h} 5=0.6$.
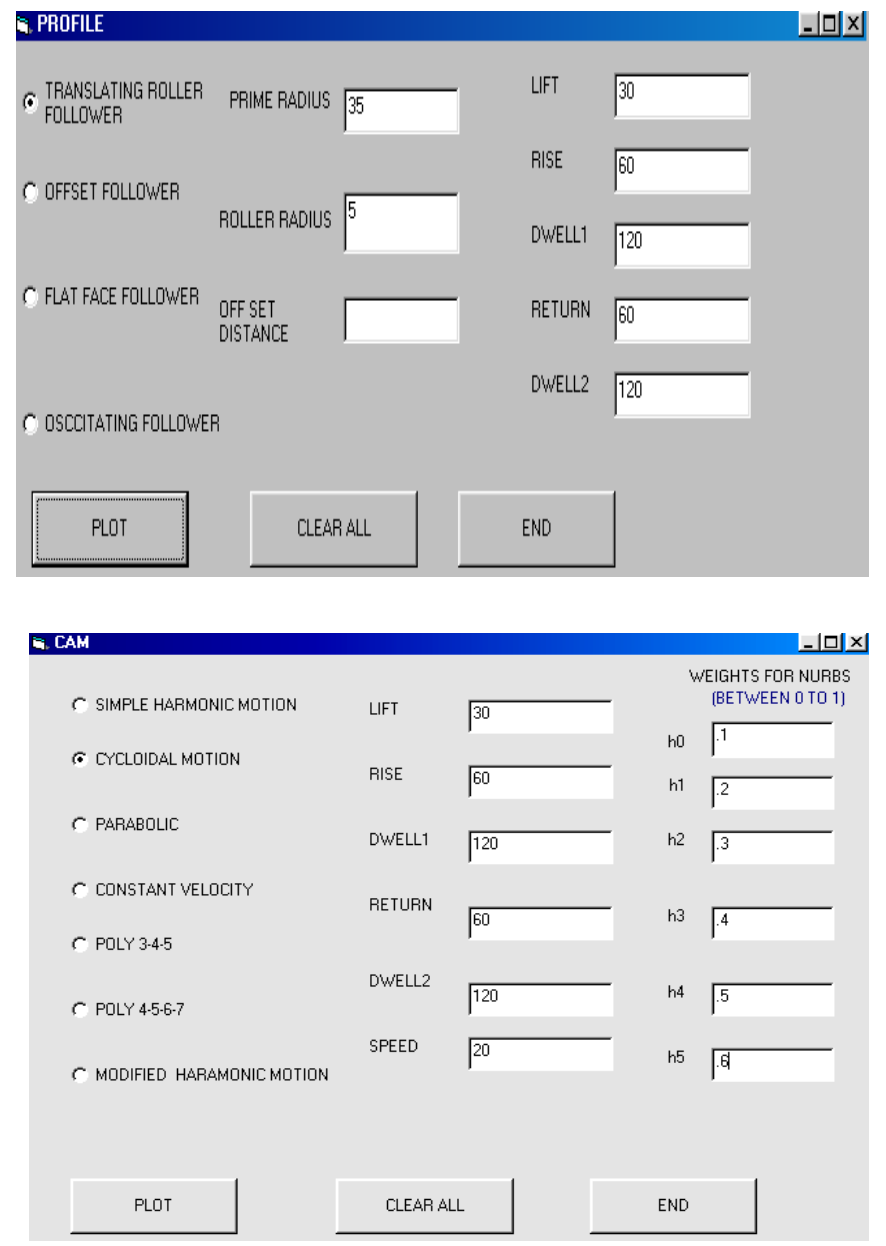

Fig.2: Input data: follower motion is cycloidal, 


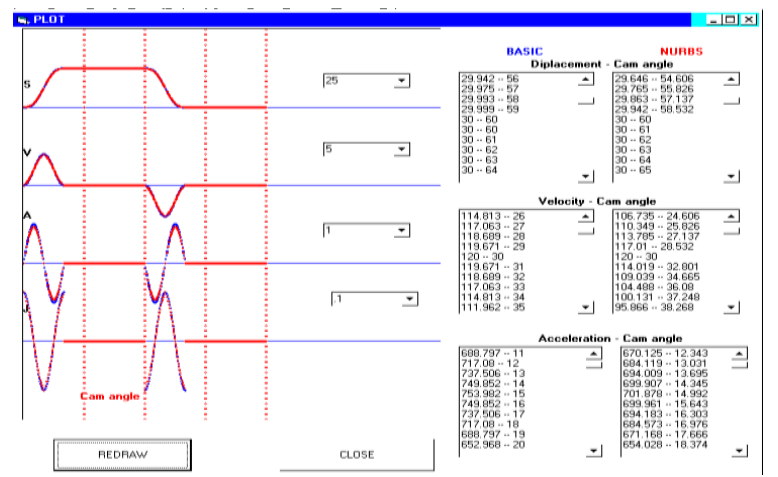

Fig.3: Graphical and numerical displacement(s), velocity (v), acceleration, jerk (j).

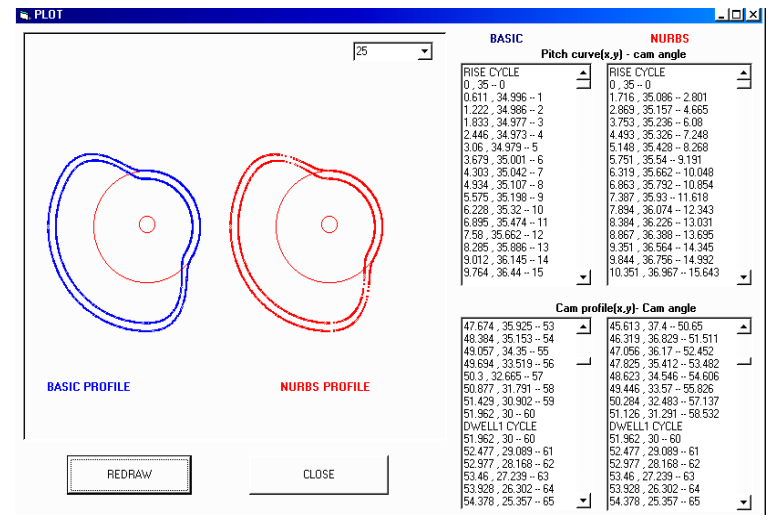

Fig 4: cam profiles and cutter location data points for CNC machining of basic curves and approximated NURBS against cam rotation angle $(\theta)$ for a typical input data.

\section{ANALYSIS OF MOTION CURVES}

Using the integrated system, an analysis was performed to determine the maximum velocities and maximum acceleration achieved by a disc-cam designed for different types of follower motions for basic curves and approximated NURBS (as shown in table 1). Lower maximum velocity and lower maximum acceleration provide low jerk, which is useful for selection of best cam profile. Using a follower displacement height of $30 \mathrm{~mm}$, the integrated system was used to compute the maximum velocities, maximum acceleration for disc cam in which the rise and return had equal angle intervals of $60^{\circ}$ and with the rise and return having the same type of motion curve and dwell of $120^{\circ}$ each. This was done for all six types of motions (SHM, Cycloidal etc) for basic curves and approximated

NURBS. Three cam speeds $5 \mathrm{rpm}, 20 \mathrm{rpm}$ and 100 rpm were used. The results in table 1 shows maximum velocities $(\mathrm{mm} / \mathrm{sec})$ and maximum acceleration $\left(\mathrm{mm} / \mathrm{sec}^{2}\right)$ for each follower motion basic curves and approximated NURBS.

From the above analysis ( Table 1 ) it clear that 4-56-7 polynomial curve has maximum acceleration and parabolic has minimum acceleration for the same cam speed among all the motion curves. Similarly 4-5-6-7 polynomial has maximum velocity and SHM has minimum velocity for the same cam speed among all the motion curves. Approximated NURBS have low maximum acceleration compared to basic curves for cycloidal, 3-4-5 polynomial, 4-5-6-7 polynomial, modified harmonic motions. So whenever minimum jerk motion (i.e. optimum motion) is required, designer can select approximated NURBS instead of basic curves for cycoidal, 3-4-5 polynomial and modified harmonic motions. This is also shown in the Figure $3 \& 4$ (for cycloidal motion).

When high velocity or high acceleration and minimum jerk required than designer can select approximated NURBS for 4-5-6-7 polynomial. When low acceleration is required designer can select either basic or approximated NURBS for parabolic motion. When low velocity is required designer can use either basic or approximated NURBS for SHM.

\section{CONCLUSION}

This paper proposes the improvement of motion characteristics of a cam-follower system by approximation of NURBS to basic curves. An integrated system is developed which provides useful design information (graphically and numerically) such as displacement, velocity, acceleration and jerk against each cam rotation for basic curves and approximated NURBS. 
Table 1. Maximum velocities $(\mathrm{mm} / \mathrm{sec})$ and maximum accelerations $\left(\mathrm{mm} / \mathrm{sec}^{2}\right)$ for different cam speeds.

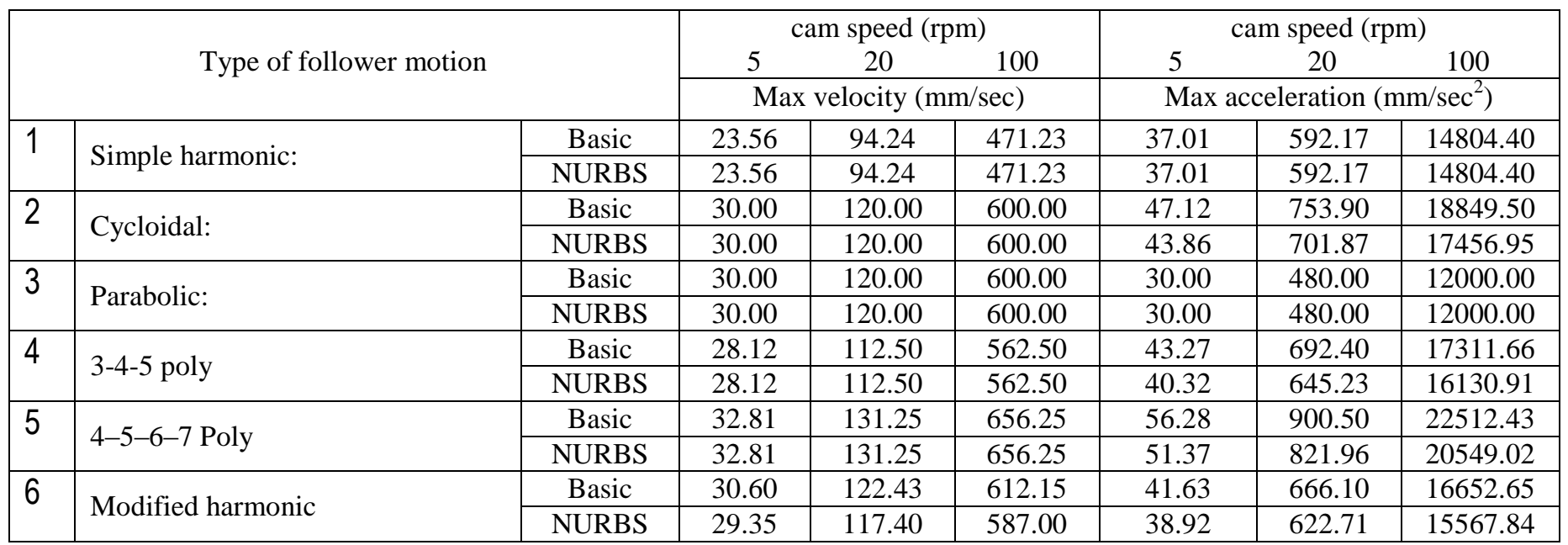

The analysis can be carried out to select best cam profile (minimum jerk) based on low maximum velocity and low maximum acceleration. The system also provides disc-cam profile coordinates to manufacture the cam on CNC machine. These numerical values are directly copied from the system to any CAM (computer aided manufacturing) software, which generates NC code automatically. So it avoids the writing NC code manually and saving in the time of manufacturing. The NC code fed to the CNC milling machine, which produces required cam profile.
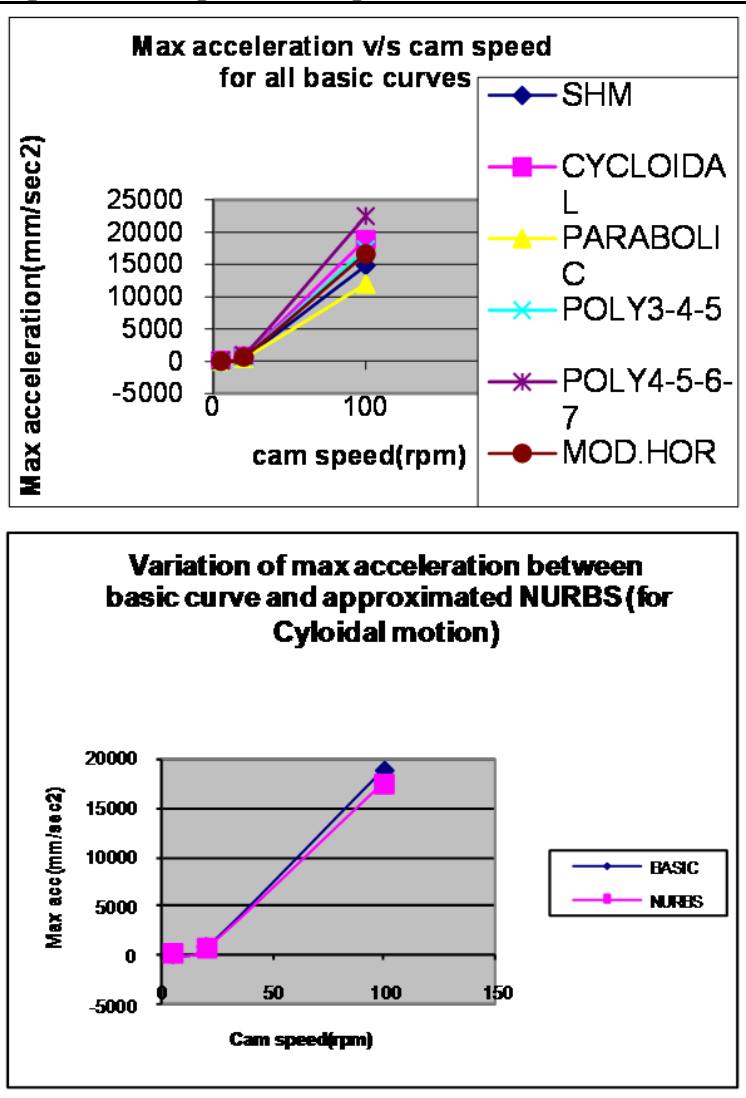

Fig.5: Graphs showing variation maximum acceleration between basic and approximated NURBS (for cycloidal motion) against cam speed.

\section{REFERENCES}

[1] P. W. Jensen, Cam Design and Manufacture, 2nd edn, Marcel Dekker, 1987.

[2] Mortenson, Geometric Modelling, John Wiley, 1985.

[3] Rothbart, H.A., 1956, Cams: Dynamics, and Accuracy, John Wiley \&Sons, New York.

[4] Ibrahim Zeid, CAD/CAM, Tata McGraw-Hill Edition 1998. Chen, F.Y., 1972, "A Refined Algorithm for Finite Difference Synthesis of Cam Profiles," Mechanism and Machine Theory, Vol. 7, pp. 453-460.

[5] Chen, F.Y., 1973," Analysis and Design of Cam-Driven Mechanisms with Nonlinearities," ADME Journal of Engineering for Industry, Vol. 95, pp. 685-694.

[6] Dhande, S. G., Bhadoria, B. S., and Chakraborty, J., 1975, "A Unified Approach to the Analytical Design of Three Dimensional Cam Mechanisms," ASME Journal of Engineering for Industry, 97B (1), pp. 327-333.

[7] Tsay, D.M., and Huey C.O. Jr., 1989, "Synthesis and Analysis of Cam-follower systems with Two degrees of freedom," ASME advances in Design Automation, Vol. 3, pp 281-288.

[8] Sadek, K., and Daadbin, A., 1990, "Improved Cam Profiles For High-Speed Machinery Using Polynomial Curve Fitting," Journal of Process Mechanical Engineering, 204, pp. 127-132.

[9] Yang, M., and Kim, C., 1994, "A CAD/CAM System for Precision Cams with Three CNC Interpolation Methods," 
Sateesh et al : Improvement in Motion Characteristics of ...

International Journal of Advanced Manufacturing Technology, Vol. 9, pp. 87-92.

[10] Chan, and Sim, S., 1996, "Optimum Cam Design," International Journal of Computer Applications in Technology, pp. 34-47.

[11] Wang, W. H., Tseng, C.H., and Tsay, C.B., 1997, "Surface Contact Analysis for a Spatial Cam Mechanism," ASME Journal of Mechanical Design, Vol. 119, pp. 169-177.

[12] Masood, S. H., and Lau, A.,1998, "A CAD/CAM System for the Machining of Precision Cams using A Half Angle Search Algorithm", International Journal of Advanced Manufacturing Technology, 14(3), pp. 180-184.
[13] Masood, H.S., 1999, "A CAD/CAM System for High Performance Precision Drum Cams," International Journal of Advanced Manufacturing Technology, pp. 32-37.

[14] Kim, J. H., Ahn, K. Y., and Kim, S. H., 2002,"Optimal Synthesis of a Spring-Actuated Cam Mechanism using a Cubic Spline", Journal of Mechanical Engineering Science,Vol. 216, pp. 875-883

[15] Sateesh, N., C.S.P Rao, and Janardhan Reddy, T.A., 2009, "Optimisation of cam-follower motions using Bsplines" "International Journal for Computer Integrated Manufacturing (IJCIM), Publisher: Taylor \& Fancis, England, Vol.22, No. 6, pp. 515-523 [16] . 